\title{
Gyrokinetic Simulations in General Geometry and Applications to Collisional Damping of Zonal Flows
}

\author{
Z. Lin, T. S. Hahm, W. W. Lee, W. M. Tang, and R. B. White \\ Princeton Plasma Physics Laboratory, Princeton University, P. O. Box 451, Princeton, NJ 08543
}

\begin{abstract}
A fully three-dimensional gyrokinetic particle code using magnetic coordinates for general geometry has been developed and applied to the investigation of zonal flows dynamics in toroidal iontemperature-gradient turbulence. Full torus simulation results support the important conclusion that turbulence-driven zonal flows significantly reduce the turbulent transport. Linear collisionless simulations for damping of an initial poloidal flow perturbation exhibit an asymptotic residual flow. The collisional damping of this residual causes the dependence of ion thermal transport on the ion-ion collision frequency even in regimes where the instabilities are collisionless.

PACS: 52.65.Tt, 52.35.Qz, 52.25.Fi
\end{abstract}

\section{INTRODUCTION}

Nonlinear gyrokinetic simulations [1] are playing an increasingly important role in understanding the physics of pressure-gradient-driven microinstabilities which are leading candidates to account for the ion thermal transport in magnetically confined fusion plasmas. With the development of a low noise nonlinear $\delta f$ algorithm [2] and the rapid increase in parallel computing power, realistic three-dimensional particle simulations of microturbulence in tokamaks have become possible in both a full torus using Cartesian coordinates [3] and a flux-tube geometry using quasiballooning coordinates [4]. The fluxtube code is based on the assumption of scale separation between fluctuation and equilibrium profiles. In the absence of equilibrium profile variations, it is not surprising that a gyro-Bohm scaling has been observed in flux-tube simulations. Therefore, the key issues of turbulent transport scaling, steep pressure profiles in transport barriers, and meso-scale physics can only be effectively studied in global simulations. The Monte-Carlo sampling of velocity phase space in the particle simulation is more efficient in multi-dimensionality than the direct discretization using phase space grids in a Vlasov simulation [5].

Previous nonlinear full torus simulations assumed a high aspect ratio axisymmetric equilibrium and neglected equilibrium profile variations (e.g., gyroradius variations across the poloidal plane). This was necessitated by the limitations of Cartesian representations and the spectral approach in solving the gyrokinetic Poisson equation. Hence, developing a general geometry capability for a nonlinear gyrokinetic code not only represents an important fundamental advance but is also of great interest for practical applications. Furthermore, transport barriers in enhanced confinement regimes are characterized by sharp pressure gradients. Cartesian coordinates are not efficient for dealing with these complicated equilibrium configurations, especially when only numerical equilib- rium data rather than analytic formulas are available. The spectral method for solving the Poisson equation is not valid in a general geometry equilibrium such as the steep pressure profile of transport barriers or the finite poloidal magnetic field of low aspect ratio machines. In this paper, magnetic coordinates [6] and a nonspectral Poisson solver [7] suitable for general geometry are utilized to address these issues.

Magnetic coordinates give the most general coordinate system for any magnetic configuration possessing nested surfaces. The property of straight field lines in these coordinates is desirable for describing the microinstabilities for which mode structures are field aligned and for efficiently treating the electron dynamics. Furthermore, the unique ability of a single code to simulate both a full poloidal cross section and an annulus box with radially periodic boundary conditions provides a connection between global simulations and flux-tube simulations. The guiding center equations of motion [8] can be derived from a Hamiltonian formulation which conserves phase space volume and is best for integrating particle orbits for a long period. The fact that only scalar field quantities are needed to calculate the guiding center orbit in this framework is most suitable for the general geometry equilibrium.

The paper is organized as follows. Section II first provides the basic formalism of this new general geometry global gyrokinetic code. Results of convergence studies are then presented. Section III discusses the application to the collisionless dynamics of turbulence-driven $\mathbf{E} \times \mathbf{B}$ zonal flows. Effects of collisional zonal flow damping on turbulent transport are studied in Section IV. Conclusions are drawn in Section V. 


\section{GYROKINETIC SIMULATIONS USING MAGNETIC COORDINATES}

\section{A. Basic formalism}

In gyrokinetic simulations, the gyrokinetic VlasovPoisson system is solved by advancing the particle position in phase space and calculating the self-consistent perturbed field recursively in a pre-defined coordinate system. In this section, magnetic coordinates, Hamiltonian guiding center equations of motion and the general geometry Poisson solver used in our gyrokinetic toroidal code (GTC) are presented along with numerical techniques for 3-dimensional gyrophase-averaging and boundary conditions for both global and annulus simulations.

In a general magnetic configuration possessing nested toroidal magnetic surfaces, the magnetic field $\mathbf{B}$ can be written [8] in the contravariant form

$$
\mathbf{B}=\frac{1}{q} \nabla \zeta \times \nabla \psi+\nabla \psi \times \nabla \theta,
$$

and in the covariant form

$$
\mathbf{B}=g \nabla \zeta+I \nabla \theta+\delta \nabla \psi
$$

The magnetic coordinates $(\psi, \theta, \zeta)$ are, respectively, toroidal flux, poloidal and toroidal angles. $g, I$ and $\delta$ are related to the poloidal, toroidal and Pfirsch-Schluter currents, respectively. $q$ is the safety factor. The Jacobian is chosen to be: $J=(g q+I) / B^{2}$. The field line is straight in these coordinates, and only scalar quantities are needed to integrate guiding center orbits. The simulation domain is discretized in a rectangular grid layout in $\left[f_{1}(\psi), f_{2}(\theta), \zeta\right]$ space. The arbitrary functions $f_{1}$ and $f_{2}$ provide flexibilities for adjusting the desired resolution and maximizing orthogonality across the poloidal plane.

The guiding center Hamiltonian is [8]:

$$
H=\frac{1}{2} \rho_{\|}^{2} B^{2}+\mu B+\bar{\Phi}
$$

where $\rho_{\|}=v_{\|} / B, \mu$ is the magnetic moment which is constant in gyrokinetic simulations, and $\bar{\Phi}$ is the gyrophaseaveraged electrostatic potential. The mass and charge of particles are normalized to unity throughout the paper. The guiding center equations in canonical form are:

$$
\begin{array}{ll}
\frac{d P_{\zeta}}{d t}=-\partial_{\zeta} H, & \frac{d \zeta}{d t}=\partial_{P_{\zeta}} H \\
\frac{d P_{\theta}}{d t}=-\partial_{\theta} H, & \frac{d \theta}{d t}=\partial_{P_{\theta}} H
\end{array}
$$

where $P_{\zeta}=g \rho_{\|}-\psi_{p}, P_{\theta}=I \rho_{\|}+\psi$, and $\psi_{p}$ is the poloidal flux. This formulation has been implemented in a guiding center code (ORBIT) [8]. The Hamiltonian approach preserves phase space volume and is most suitable for describing the long time behavior of particle orbits.

The gyrokinetic Poisson equation [1] takes the following form,

$$
\frac{\tau}{\lambda_{D}^{2}}(\Phi-\tilde{\Phi})=4 \pi e\left(\delta \bar{n}_{i}-\delta n_{e}\right)
$$

where

$$
\tilde{\Phi}(\mathbf{x})=\frac{1}{2 \pi} \int \bar{\Phi}(\mathbf{R}) F_{M}\left(\mathbf{R}, \mu, v_{\|}\right) \delta(\mathbf{R}-\mathbf{x}+\rho) d \mathbf{R} d \mu d v_{\|} d \phi
$$

and

$$
\bar{\Phi}(\mathbf{R})=\frac{1}{2 \pi} \int \Phi(\mathbf{x}) \delta(\mathbf{x}-\mathbf{R}-\rho) d \mathbf{x} d \phi
$$

Here $\mathbf{x}$ and $\mathbf{R}$ are particle and guiding center positions, respectively. $\rho$ is the gyroradius and $\phi$ is the gyrophase. $\tau=T_{e} / T_{i}$ and $\lambda_{D}$ is the plasma Debye length. $\delta \bar{n}_{i}$ and $\delta n_{e}$ are the ion guiding center and electron perturbed density response, respectively. The term of $(\Phi-\tilde{\Phi})$ represents the ion polarization density. It can be shown $[9,10]$ that a set of gyrokinetic Vlasov equations corresponding to the guiding center equation and Eq. (1) conserves the energy. Transformation between $\tilde{\Phi}$ and $\Phi$ must be carried out in order to solve Eq. 1. In a global code using Cartesian coordinates, this is usual done by transforming Eq. 1 to the Fourier space with some approximations such as zero poloidal field and constant gyroradius across the poloidal plane. Here, we utilize a generalized gyrokinetic Poisson solver [7] which is based on the physical process of gyrophase-averaging and employs local operations in configuration space to compute the polarization density response. Specifically, the integrations over gyrophase $\phi$ in Eq. 2 and Eq. 3 are carried out by a numerical averaging along a charged ring which corresponds to the particle gyro-orbit [1]. This method automatically takes into account the profile variation effects and is suitable for general geometry. Its local operations in configuration space are also amenable to massively parallel algorithms.

Previous procedures of the gyrophase-averaging have neglected the poloidal field so that the gyro-orbit is in the poloidal plane. However, in a low aspect ratio device, the poloidal field can be comparable to the toroidal field and the gyrophase-averaging should be along the true gyro-orbit which is in a plane perpendicular to the magnetic field. In magnetic coordinates, we can project the gyro-orbit back onto the poloidal plane by following the field line. The gyro-averaging can then still be done on the poloidal plane along the projected ecliptic ring. This procedure is valid for $k_{\|} \rho_{i} \ll 1$ which holds for microturbulence and can reduce a 3 -D gyro-averaging to a 2-D operation even in the situation of non negligible poloidal field. The grid spacing in the toroidal direction is only limited by the shortest wavelength of interest. 
The flexibility of magnetic coordinates enables us to simulate both a full poloidal cross section and an annular box $\left[\psi_{1}, \psi_{2}\right]$ with a variety of boundary conditions including the radially periodic boundary condition. This unique capability allows a connection between global simulations and flux-tube simulations. The perturbed electrostatic potential is set to zero at the wall in all global simulations. Here, we present a radially periodic boundary condition similar to that of the flux-tube simulation $[11,4]$. Defining a new variable $\alpha=\zeta / q-\theta$, we can use $\alpha$ to label the magnetic field line and $\theta$ to measure the parallel distance along the flux tube. The periodic boundary condition in the radial direction is defined as $\Phi\left(\psi_{1}\right)=\Phi\left(\psi_{2}\right)$ with $\theta$ and $\alpha$ kept constant. Specifically, we impose periodic boundary conditions such that

$$
\Phi\left(\psi_{1}, \theta_{1}, \zeta_{1}\right)=\Phi\left(\psi_{2}, \theta_{2}, \zeta_{2}\right)
$$

with

$$
\theta_{2}=\theta_{1} \quad \text { and } \quad \zeta_{2}-\zeta_{1}=\left(q_{2}-q_{1}\right) \theta_{1}
$$

Here, we use subscripts 1 and 2 to label quantities at the inner and outer boundaries, respectively. Since the field line maps the whole annulus, the physical periodic boundary condition of $\Phi(\theta)=\Phi(\theta+2 \pi)$ requires,

$$
q_{2}-q_{1}=\text { integer. }
$$

\section{B. Convergence studies}

Both linear and nonlinear simulations have been carried out to rigorously benchmark the GTC code against earlier analytic and computational models. Convergence studies have also been performed in nonlinear simulations using a representative tokamak core plasma parameters. In the core region of this plasma, the temperature gradient peaks at a radial location of $r=0.5 a$ where $a$ is the minor radius. The dimensionless plasma parameters used in simulations are: $R_{0} / L_{T}=6.9, \eta_{i} \equiv L_{n} / L_{T}=3.2$, $q=1.4, \hat{s} \equiv(r / q)(d q / d r)=0.78, T_{e} / T_{i}=1$, and $\epsilon \equiv a / R_{0}=0.36$. Here $R_{0}$ is the major radius, $L_{T}$ and $L_{n}$ are the temperature and density gradient scale lengths, respectively. $T_{i}$ is the ion temperature and $T_{e}$ is the electron temperature. The size of the plasma column is $a=160 \rho_{i}$ where $\rho_{i}$ is the thermal ion gyroradius measured at $r=0.5 a$. The simplified model used in this section includes: parabolic $q$ profile, circular cross section, and adiabatic electron response with no turbulence driven $\mathbf{E} \times \mathbf{B}$ flow. Ion-temperature-gradient (ITG) modes are found to be unstable with these parameters. The simulation domain is limited to the region of $0.25<r / a<0.75$ in this study with fixed boundary conditions and a narrow profile of temperature gradient. Convergence studies with respect to size of time step, number of grid points and number of particles in nonlinear simulations have been carried out. In particular, convergence of the ion heat conductivity $\chi_{i}$ and fluctuation energy level with respect to the number of particles is shown in Fig. 1. A total of 20 million grid points, i.e., $(80 \times 640 \times 384)$ are used in the $(\psi, \theta, \zeta)$ space. The ion heat conductivity remains unchanged when the number of particles is increased from 32 million to 80 million. This indicates that $\chi_{i}$ is well converged. The difference in field energy with different number of particles as shown in Fig. 1 is due to short wavelength modes which need more particles to resolve and have little effect on transport. These short wavelength modes can also be suppressed by numerical filtering.

In Fig. 1, both $\chi_{i}$ and fluctuation energy initially grow exponentially in the linear phase, then peak in the quasilinear stage, and finally drop to the much lower steady state values in the fully developed nonlinear phase. This behavior has been observed in all previous gyrokinetic particle simulations. Questions have been raised regarding these steep drops. We found that the transport at the quasilinear peak is caused by a few linearly most unstable modes with $k_{\theta} \rho_{i} \sim 0.4$ which grow to very high amplitudes. The fluctuation energy is then transferred from these linearly dominant modes to the longer wavelength modes with $k_{\theta} \rho_{i} \sim 0.2$ which have smaller amplitude and are responsible for the steady state transport. This process is visualized in the time history of individual modes as shown in Fig. 2.

The issue of discrete particle noise has been examined since the early days of particle simulations. With the improvement in theory and numerical algorithm, and the rapid increase in computer power, the particle noise in simulations has been dramatically reduced and is believed not to have large effects in nonlinear dynamics. The mode history of Fig. 2 indicates that very small, if any, particle noise exists in the nonlinear phase. It should be emphasized that such a good numerical property has only been captured in the straight-field-line magnetic coordinates which can better represent the fluctuation structure. Another interesting feature of Fig. 2 is the very coherent structure of individual mode history even though millions of modes are coupled together in the turbulent system.

\section{DYNAMICS OF FLUCTUATION-DRIVEN $\mathrm{E} \times \mathrm{B}$ ZONAL FLOWS}

$\mathbf{E} \times \mathbf{B}$ flow shear suppression of turbulence is the most likely mechanism to be responsible for various forms of confinement enhancement. Recent experimental data show evidence of $\mathbf{E} \times \mathbf{B}$ flows [12] which cannot be explained by neoclassical theory. These flows can be driven by turbulence itself [13-15], and mainly in the poloidal direction for high aspect ratio devices. They are referred to 
as the radial modes, $(0,0)$ modes or zonal flows [16], and have been found to play an important role in regulating nonlinear saturation and turbulent transport in flux-tube gyrofluid [11,17] and gyrokinetic [4] simulations.

\section{A. Linear damping of zonal flows}

In toroidal geometry, an initial source of zonal flows is damped by the collisionless transit time magnetic pumping effects [18]. A residual flow can survive this linear collisionless damping process [19], and play a key role in determining the turbulent transport level in nonlinear simulations, thus its long time behavior must be treated accurately. An illuminating test problem of predicting the residual flow level in response to an initial flow perturbation introduced by a flux-surface-averaged charge separation has been solved analytically [19]. In our simulation, the relaxation of the perturbed poloidal flow, shown in Fig. 3, occurs initially through a fast magnetic pumping process and is then followed by a slowly damped oscillation with a characteristic frequency corresponding to that of the geodesic acoustic mode (GAM) [20]. The residual levels of poloidal flow measured from simulations, shown in Fig. 4, agree with theoretical results in the high aspect ratio limit where the theory is valid. We note that similar results have been obtained in a fluxtube gyrokinetic code [21]. In the damping processes, the $m=1$ harmonic can be excited by toroidal coupling to the $m=0$ harmonic. When the safety factor $q$ is large, the GAM oscillations persist as the $m=1$ harmonic becomes undamped [22].

Since the microturbulence intensity in tokamaks typically has an in-out variation, it is important to be able to address the generation of poloidal flows from a $m=1$ source due to toroidal couplings. Because of the up-down symmetry of the equilibrium magnetic field, only that part of the $m=1$ harmonic that is even in $\theta$ can generate poloidal flow. This has been verified by simulation results (Fig. 5) which show that the time asymptotic poloidal flow is nonzero for an even- $\theta$ source and zero for an odd $-\theta$ source. The flow generation results from trapped particle dynamics which has not been accurately described by fluid simulations.

\section{B. Nonlinear generation of zonal flows and effects on turbulence}

Previous studies using global gyrokinetic simulations [3] either artificially suppress zonal flows or found only a small reduction in ion thermal transport at nonlinearly saturated state when shear flows effects were included in the nonlinear evolution. Moreover, $\mathbf{E} \times \mathbf{B}$ flows with scale length of the system size have been generated in global codes, while in flux-tube codes fluctuating zonal flows with a radial characteristic scale length comparable to that of the ambient turbulence have been the dominant feature. Finally, global codes typically yields a much lower value of $\chi_{i}$ than that from flux-tube codes. To study the origin of these different results, we have carried out nonlinear simulations with either the whole cross-section or an annulus geometry in the same code. The annulus simulation model is designed to capture the key features of local simulations, i.e., uniform pressure gradient profile and radially periodic boundary conditions described in the previous section. In global simulations, the pressure gradient is nonuniform and a fixed radial boundary condition is used. In order to find out the relative importance of pressure profile and the radial boundary condition in causing the different results, global simulations are carried out with either a narrow profile of pressure gradient or a broad profile of pressure gradient which is similar to that of the annulus simulations. The same plasma parameters specified in the previous section are used in these simulations.

Our simulations [23] recover the previous results from the annulus simulations and the results from the global simulations with a narrow profile of the pressure gradient. While fine scale structure of flows are dominant in annulus simulations, a single-well structure in $\mathbf{E} \times \mathbf{B}$ flows emerges in the global simulations with a narrow profile. In addition, the ion heat conductivity from annulus simulations is much higher than that from the global simulations. When we make the profile of the pressure gradient broad in global simulations, the fine scale components of $\mathbf{E} \times \mathbf{B}$ flows with radial characteristic scale length comparable to the turbulence decorrelation length become dominant. Good agreement in both the structure of $\mathbf{E} \times \mathbf{B}$ flows and ion heat conductivities are obtained between annulus and global simulations with broad pressure gradient profile. These agreements indicate that the radially periodic boundary condition in local codes is not responsible for the results differing from global code results, at least for the cases we have studied. Therefore the narrow profile of pressure gradient in global codes causes the results to differ from the local code results.

Quantitative difference in $\chi_{i}$ exists between our annulus simulation model and flux-tube gyrokinetic model [4]. While our annulus simulations are designed to capture the key features of the local simulations, some differences remain between them. The variations of safety factor and aspect ratio are kept in the annulus code, while the flux-tube code has constant aspect ratio and connection length (keeping magnetic shear) in radius. The other difference is that annulus code simulates the whole flux surface with all toroidal mode numbers while flux-tube code simulates a fraction of the flux surface with a selected subgroup of toroidal modes.

We have also carried out nonlinear simulations with the $\mathbf{E} \times \mathbf{B}$ flows suppressed for all these three cases. It 
is found that a significant reduction of ion heat conductivity occurs when the turbulence-generated $\mathbf{E} \times \mathbf{B}$ flows are retained in nonlinear global simulations. The key mechanism of reducing transport by the $\mathbf{E} \times \mathbf{B}$ flows is the breaking of turbulence eddies, and consequently the reduction of the radial decorrelation length and fluctuation levels [24,25]. Another important effect of the turbulence-generated $\mathbf{E} \times \mathbf{B}$ flows appears in the individual mode history. When $\mathbf{E} \times \mathbf{B}$ flows are suppressed, for fixed $k_{\theta} \rho_{i}$, the potential exhibit regular oscillation with a constant phase (Fig. 2 in previous section). That coherent mode history corresponds to a frequency spectrum with a well-defined peak which may indicate that the mode coupling is not so strong and that the nonlinear wave-particle interaction may be more important than previously expected. When $\mathbf{E} \times \mathbf{B}$ flows are included in nonlinear simulations, the oscillation becomes chaotic with no regular phase relation.

\section{COLLISIONAL DAMPING OF ZONAL FLOWS}

The residual flow observed in collisionless simulation is eventually damped out by ion-ion collisions and possibly, by nonlinear effects. In the present work, the collisional decay of the flow perturbation in the linear gyrokinetic simulation agrees well with the analytical calculation [26]. The effective damping time measured from the simulation is very close to the theoretical prediction of $\tau_{d}=1.5 \epsilon \tau_{i i}$, where $\epsilon \equiv r / R_{0}$ is the local inverse aspect ratio and $\tau_{i i}$ is the ion-ion collision time. In full torus nonlinear ITG simulations, we use a momentum and energy conserving Fokker-Planck operator which has been rigorously benchmarked for neoclassical transport [27]. These simulations used plasma parameters in Section II with fixed boundary conditions of $\phi=0$ enforced at $r<0.1 a$ and $r>0.9 a$, a pressure gradient profile of $\exp \left\{-[(r-0.5 a) / 0.3 a]^{6}\right\}$, and an adiabatic electron response [28] with $\delta n_{e} / n_{0}=e(\phi-\langle\phi\rangle) / T_{e}$, where $\langle\cdots\rangle$ represents the flux surface average. In each nonlinear simulation, we calculated 10000 time steps of $8 \omega_{c i}$ for the trajectories of 84 million gyrocenter centers interacting with the self-consistent turbulent field, which was discretized by 21 million grid points. ITG mode has a linear threshold [21] of $R_{0} / L_{T}=4$. We scan $R_{0} / L_{T}$ in the present studies to assess sensitivity of the collisional effects on the proximity to ITG marginality.

For a strong ITG drive of $R_{0} / L_{T}=6.9$, both turbulence and zonal flows saturate and reach steady state in collisionless simulations. The fact that zonal flows saturate even without collisional damping indicates that strong turbulence can also damp zonal flows possibly through nonlinear effects. When the realistic ion-ion collisions with $\nu^{*}=0.045$ are included in the simulation, the steady state ion heat conductivity $\chi_{i}$ is increased by about one half from the collisionless value. The saturated zonal flow level decreases due to collisions. Meanwhile, the change in linear growth rate is negligible. Furthermore, when zonal flows are not included in the simulations, collisions have little effects on the turbulence transport. We therefore conclude that the enhancement of transport by collisions in the presence of zonal flows is through the neoclassical damping of zonal flows.

When turbulence drive $R / L_{T}$ is reduced, nonlinear flow damping becomes insignificant. The collisionless system can undergo a transition to a flow-dominated state where zonal flows generated from the initial growth of turbulence completely suppress the turbulence and transport [14,29]. In this paper, we characterize this regime as the weak turbulent regime. Collisionless nonlinear flux-tube gyrokinetic simulations of the same plasma parameters have found [21] that, for $R / L_{T}=4$ to 6 , no transport is produced even though the system is linearly unstable to ITG modes. However, collisions can remove this nonlinear upshift of critical gradient by slowly damping zonal flows. The ion thermal transport level will then strongly depend on the collision frequency. We now reduce the turbulence drive to $R / L_{T}=5.3 \mathrm{in}$ our nonlinear simulations. This is not the generic ITG turbulence regime, but is suitable for the purpose of this paper. We scan the ion-ion collision frequency upward from experimental value to facilitate studies of the long term collisional effects on the zonal flow dynamics. In the collisionless simulation, the saturated heat flux decreases to insignificant level due to shearing effects of zonal flows which reach a steady state level. When collisions are introduced, zonal flows are damped and a finite transport level is obtained. As we increase collision frequency without changing other parameters, we observe the increase of time-averaged $\chi_{i}$ without a tendency toward a saturation for the whole scan range of collision frequency, which is up to 8 times the experimental value. The ion heat conductivity sensitively depends on the collision frequency in this weak turbulence regime [30]. This collisionality-dependence of the turbulent transport implies that an accurate treatment of the linear poloidal flow damping $[19,26]$ in nonlinear simulation codes is essential in predicting transport level.

The simulation time history for the case of $\nu^{*}=0.17$ is shown in Fig. 6. The ITG instabilities evolve from a linear phase of exponential growth to a nonlinear stage in which zonal flows are generated. When the effective shearing rate [31], or root mean square shearing rate [14] of zonal flows exceeds the ambient turbulence decorrelation rate which can be approximated by the ITG linear growth rate [17], the ITG turbulence and associated transport are significantly reduced. Zonal flows are then slowly damped by the ion-ion collisions and become weaker. When the effective shearing rate is below the growth rate, the ITG turbulence grows again and drives zonal flows. These turbulence-zonal flows interactions 
modulated by collisions result in a cyclic, bursting behavior of fluctuations, transport and zonal flows. From a scan of the collision frequency, we observe that the bursting period is of the order of the collisional damping time of the zonal flows, $\tau_{d}$. The oscillation of zonal flows lags behind that of fluctuation by a phase of $90^{\circ}$, an indication of the causal relation between the turbulence and zonal flows.

\section{CONCLUSION}

A fully three-dimensional gyrokinetic particle code using magnetic coordinates for general geometry has been developed and applied to the investigation of ion-temperature-gradient turbulence in toroidal geometry. Our results yield the important conclusion that turbulence-driven $\mathbf{E} \times \mathbf{B}$ zonal flows can significantly reduce the turbulent transport. Nonlinear simulations show that the turbulent transport increases with ion-ion collision frequency because of the neoclassical damping of zonal flows which regulate the turbulence.

\section{ACKNOWLEDGMENTS}

We acknowledge useful conversations with L. Chen and P. H. Diamond. Work supported by Department of Energy Contract No. DE-AC02-76CH03073 and in part by the Numerical Tokamak Turbulence Project.

[1] W. W. Lee, Phys. Fluids 26, 556 (1983); W. W. Lee, J. Comput. Phys. 72, 243 (1987).

[2] A. M. Dimits and W. W. Lee, J. Comput. Phys. 107, 309 (1993); S. E. Parker and W. W. Lee, Phys. Fluids B 5, 77 (1993); G. Hu and J. A. Krommes, Phys. Plasmas 1, 863 (1994); M. Kotschenreuther, Bull. Am. Phys. Soc. 34, 2107 (1988).

[3] S. E. Parker, W. W. Lee, and R. A. Santoro, Phys. Rev. Lett. 71, 2042 (1993); J. C. Cummings, PhD dissertation, Princeton University, 1995; R. D. Sydora, V. K. Decyk, and J. M. Dawson, Plasma Phys. Controlled Fusion 38, A281 (1996); Y. Kishimoto, T. Tajima, M. J. Lebrun, W. Horton, and J. Y. Kim, Phys. Plasmas 3, 1289 (1996).

[4] A. M. Dimits, T. J. Williams, J. A. Byers, and B. I. Cohen, Phys. Rev. Lett. 77, 71 (1996).

[5] J. Denavit and W. L. Kruer Phys. Fluids 14, 1782 (1981).

[6] A. H. Boozer, Phys. Fluids 24, 1999 (1981).

[7] Z. Lin and W. W. Lee, Phys. Rev. E 52, 5646 (1995).

[8] R. B. White and M. S. Chance, Phys. Fluids 27, 2455 (1984); R. B. White, Phys. Fluids B 2, 845 (1990).

[9] T. S. Hahm, Phys. Fluids 31, 2670 (1988).
[10] E. A. Frieman and L. Chen, Phys. Fluids 25, 502 (1982).

[11] M. A. Beer, PhD dissertation, Princeton University, 1995.

[12] R. E. Bell and et al, Phys. Rev. Lett. 81, 1429 (1998); R. J. Groebner, Phys. Fluids B 5, 2343 (1993).

[13] P. H. Diamond and Y. B. Kim, Phys. Fluids B 3, 1626 (1991).

[14] P. H. Diamond et al., in Proceedings of the 17th IAEA Fusion Energy Conference (IAEA-CN-69/TH3/1,1998).

[15] Liu Chen, Zhihong Lin, and Roscoe White, Excitation of Zonal Flow by Drift Waves in Toroidal Plasmas, to appear in Phys. Rev. Lett., 2000.

[16] A. Hasegawa, C. G. Maclennan, and Y. Kodama, Phys. Fluids 22, 2122 (1979).

[17] R. E. Waltz, G. D. Kerbel, J. Milovich, Phys. Plasmas 1, 2229 (1994).

[18] T. H. Stix, Phys. Fluids 16, 1260 (1973).

[19] M. N. Rosenbluth and F. L. Hinton, Phys. Rev. Lett. 80, 724 (1998).

[20] N. Winsor, J. L. Johnson, and J. M. Dawson, Phys. Fluids 11, 2448 (1968).

[21] A. M. Dimits et al., Comparisions and Physics Basis of Tokamak Transport Models and Turbulence Simulaion, to appear in Phys. Plasmas, 2000.

[22] V. B. Lebedev, P. N. Yushmanov, P. H. Diamond, S. V. Novakovskii, and A. I. Smolyakov, Phys. Plasmas 3, 3023 (1996); S. V. Novakovskii, C. S. Liu, and R. Z. Sagdeev, Phys. Plasmas 4, 4272 (1997).

[23] Z. Lin, T. S. Hahm, W. W. Lee, W. M. Tang, and R. B. White, Science 281, 1835 (1998).

[24] H. Biglari, P. H. Diamond, and P. W. Terry, Phys. Fluids B 2, 1 (1990).

[25] T. S. Hahm and K. H. Burrell, Phys. Fluids 2, 1648 (1995).

[26] F. L. Hinton and M. N. Rosenbluth, Plasma Phys. Controlled Fusion 41, A653 (1999).

[27] Z. Lin, W. M. Tang, and W. W. Lee, Phys. Rev. Lett. 78, 456 (1997).

[28] G. W. Hammett et al., Plasma Phys. Contr. Fusion 35, 973 (1993).

[29] G. Hu and W. Horton, Phys. Plasmas 4, 3262 (1997).

[30] Z. Lin, T. S. Hahm, W. W. Lee, W. M. Tang, and P. H. Diamond Phys. Rev. Lett. 83, 3645 (1999).

[31] T. S. Hahm et al., Phys. Plasmas 6, 922 (1999). 

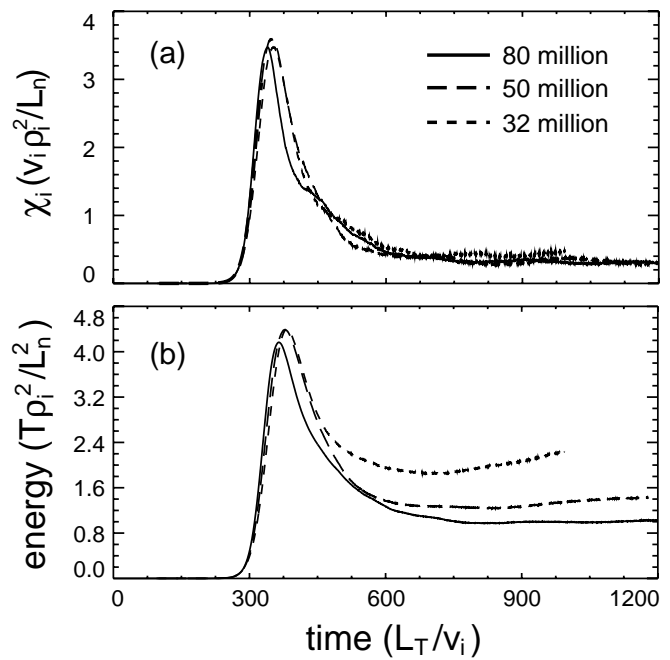

FIG. 1. Time history of ion heat conductivity $(a)$ and fluctuation energy $(b)$ in nonlinear simulations.

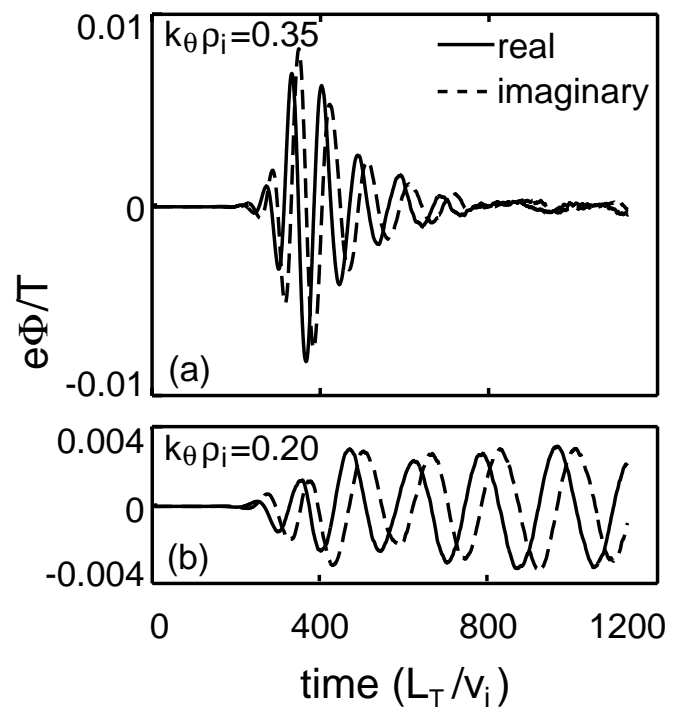

FIG. 2. Time history of a linearly dominant mode $(a)$ and a nonlinearly dominant mode $(b)$ in nonlinear simulations.

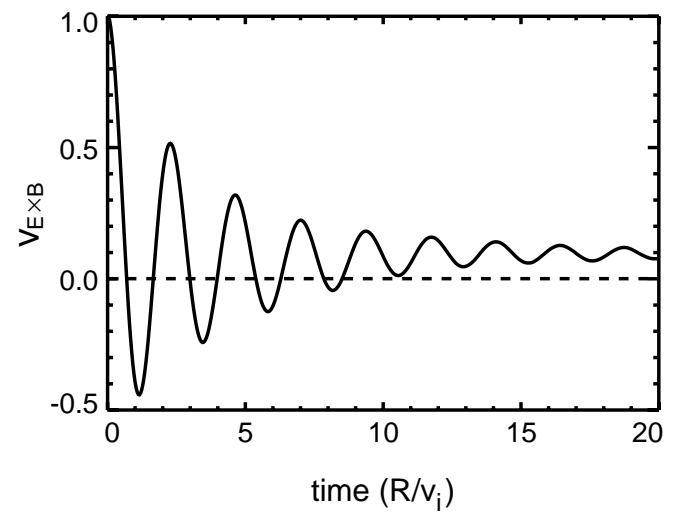

FIG. 3. Time evolution (solid line) of $\mathbf{E} \times \mathbf{B}$ flows (normalized to initial value). Dashed line is the zero level.

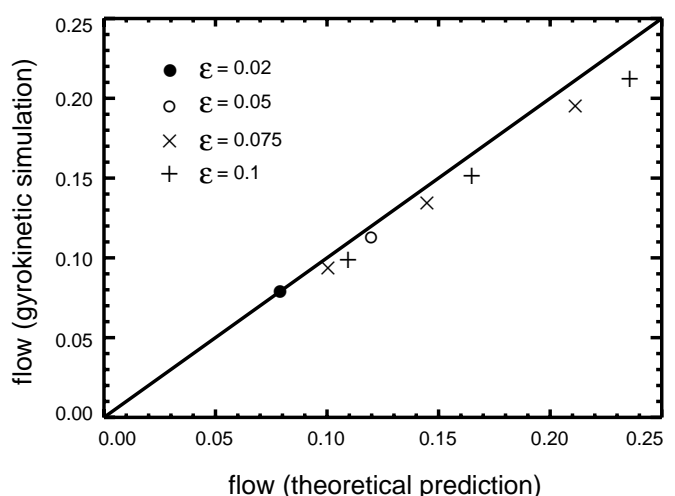

FIG. 4. Residual levels $1 /\left(1+1.6 q^{2} / \sqrt{\epsilon}\right)$ of perturbed $\mathbf{E} \times \mathbf{B}$ flows. $1<q<2$ and $k_{r} p_{i}<0.1$ in all cases.

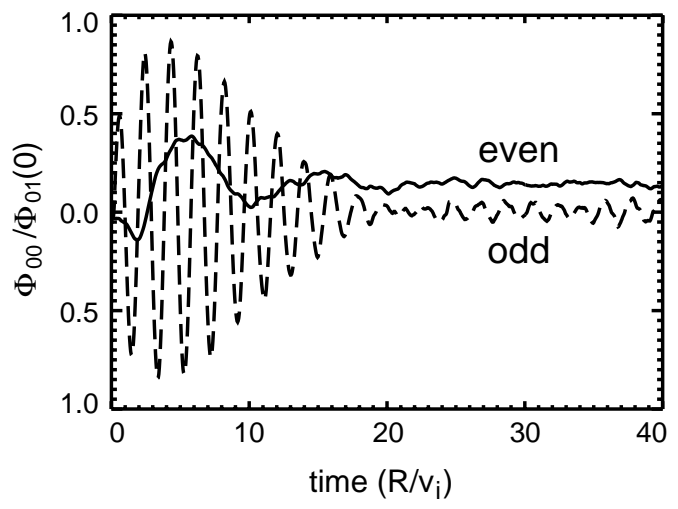

FIG. 5. Generation of poloidal flows by the $m=1$ harmonic. The initial perturbation at $t=0$ is even in $\theta$ for the solid line and odd in $\theta$ for the dashed line.

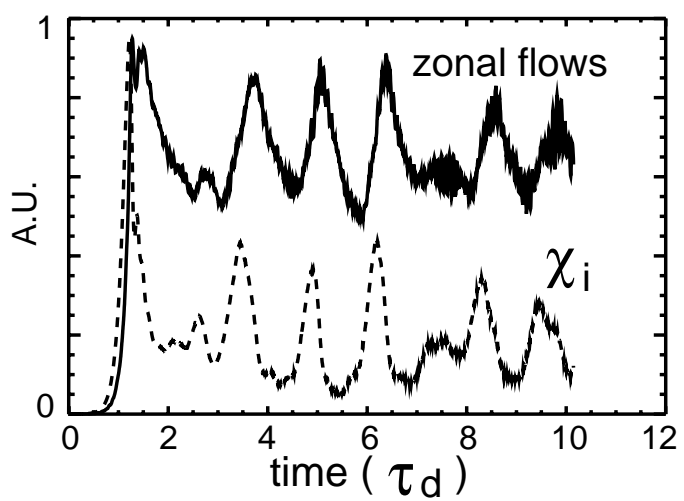

FIG. 6. Time history in nonlinear gyrokinetic simulations for zonal flow amplitude $\langle\phi\rangle$ (solid line) and ion heat conductivity $\chi_{i}$ (dotted line). 